\title{
CONTENIDO, DISTRIBUCIÓN Y ORIGEN DE HIDROCARBUROS EN SEDIMENTOS DE TRES LAGUNAS URBANAS DE CONCEPCIÓN - CHILE
}

Elizabeth González Sepúlveda*

Departamento de Química Ambiental, Facultad de Ciencias, Universidad Católica de la Santísima Concepción, Casilla 297, Concepción, Chile

Rodrigo Loyola Sepúlveda

Departamento de Oceanografía Química, Universidad de Concepción, Concepción, Chile

José Neira Hinojosa

Departamento de Análisis Instrumental, Facultad de Farmacia, Universidad de Concepción, Concepción, Chile

Felipe Neira González

Departamento de Ingeniería Industrial, Facultad de Ingeniería, Universidad de Concepción, Concepción, Chile

Recebido em 14/8/12; aceito em 20/11/12; publicado na web em 28/2/13

\begin{abstract}
CONTENT, DISTRIBUTION AND ORIGIN OF HYDROCARBONS IN SEDIMENTS FROM THREE URBAN LAGOONS IN CONCEPCIÓN - CHILE. Aliphatic hydrocarbon content, distribution and origin in superficial and deep sediments of three interconnected urban lagoons located in Concepción-Chile were studied. In all lagoons, the analysis showed that aliphatic hydrocarbons present were of biogenic and anthropogenic origin, and $n$-alkanes were predominantly from odd carbon, confirmed by a relatively high Pristane/Phytane ratio. The most abundant Polycyclic Aromatic Hydrocarbons (PAHs) were two high-molecular weight species (Fluoranthene and Pyrene) whose presence may be related with the regular activities of the area as well as with combustion processes.
\end{abstract}

Keywords: hydrocarbons; PAHs; sediments.

\section{INTRODUCCIÓN}

La determinación y cuantificación de hidrocarburos alifáticos y aromáticos policíclicos (HAPs), en sedimentos, agua y organismos, es importante para precisar el origen de este tipo de compuestos ampliamente distribuidos en el medioambiente. ${ }^{1}$ Las cuencas lacustres y márgenes continentales son particularmente favorables para la acumulación de estos compuestos.

Las lagunas y los humedales son primordiales como reguladores del balance hídrico local, reservas estacionales de agua dulce ${ }^{2}$ y sistema de regadío, y como refugio de flora y fauna local y migratoria. En Concepción - Chile existe una diversidad de lagunas y humedales urbanos sometidos a una gran presión por contaminación de diverso origen, ${ }^{3}$ por esta razón se ha hecho necesario contar con estudios que permitan una mejor planificación en su uso, manejo y conservación.

La fuente de origen de hidrocarburos alifáticos puede ser petrogénico o biogénico. Para establecer su procedencia se utilizan índices como el Índice de Preferencia de Carbono (CPI) ${ }^{4}$ las relaciones Pristano $/ n-\mathrm{C}_{17}$ y Fitano $/ n-\mathrm{C}_{18}$ que se usan a menudo para indicar el grado de degradación microbiana y la relación Pristano/Fitano (valores próximos a la unidad indica origen petrogénico).

Los HAPs, son compuestos cancerígenos de alta persistencia y riesgo ambiental. Sus principales fuentes incluyen emisiones de la quema de carbón, leña o basura; incendios forestales; emisiones de automóviles y plantas generadoras de energía. ${ }^{5}$ Debido a su origen los HAPs pueden ser diagénicos, pirogénicos, biogénicos o petrogénicos. En la última década se han desarrollado estudios ambientales forenses para analizar la presencia de determinados hidrocarburos y sus concentraciones para determinar su origen. ${ }^{5}$ Los HAPs petrogénicos, son los que están relacionados con el petróleo crudo y subproductos de éste (Naftaleno, Fluoreno, Fenantrenos, Dibenzotiofenos y Crisenos).

*e-mail: eligonza@ucsc.cl
Los HAPs pirogénicos (Fluoranteno y el Pireno son usualmente los más abundantes) son generados por la combustión de combustibles fósiles o material orgánico reciente, como la madera o cualquier combustible. Es muy probable que el tipo y calidad de la materia orgánica que se quema influya en el tipo de HAP generado. ${ }^{6}$ Algunos índices ambientales desarrollados como una forma de discriminar entre la procedencia petrogénica o antropogénica de los HAPs son las relaciones Fenantreno/Antraceno y Fluoranteno/Pireno. ${ }^{7}$

Para la identificación de la madurez de la materia orgánica se emplean diversos parámetros geoquímicos: ${ }^{8,9}$ la relación Pristano/ Fitano se utiliza para determinar si la materia orgánica es de origen continental (ambiente oxidante) o de origen marino (ambiente reductor); la relación entre hidrocarburos de bajo peso molecular a los de alto peso molecular (LMW/HMW), el cual indica si el origen es debido a algas, plancton, petróleo crudo o bacterias sedimentarias, animales marinos y sedimentos; la relación $\Sigma n$-alcanos $/ n-\mathrm{C}_{16}{ }^{10}$ indica si el origen es debido a material biogénico o petróleo; las relaciones de Pristano $/ n-\mathrm{C}_{17}$, Fitano $/ n-\mathrm{C}_{18}$; Índice de Preferencia de Carbono $(\mathrm{CPI})^{11}$ e Índice de Predominacia Impar-Par (OEP), ${ }^{12}$ proporcionan información referente a la madurez de la materia orgánica, estas dos últimas se expresan como:

$$
\begin{gathered}
\mathrm{CPI}=1 / 2 \Sigma\left[\left(\mathrm{C}_{25}-\mathrm{C}_{33}\right) /\left(\mathrm{C}_{24}-\mathrm{C}_{32}\right)+\left(\mathrm{C}_{25}-\mathrm{C}_{33} /\left(\mathrm{C}_{26}-\mathrm{C}_{34}\right)\right]\right. \\
\mathrm{OEP}=\left[\mathrm{C}_{27}+6\left(\mathrm{C}_{29}+\mathrm{C}_{31}\right) / 4\left(\mathrm{C}_{28}+\mathrm{C}_{30}\right)\right]
\end{gathered}
$$

Otro índice para determinar la fuente de hidrocarburos es la razón entre material terrígeno a acuático (TAR), propuesta por Bourbonniere et al. ${ }^{13}$ y definida como:

$$
\mathrm{TAR}=\left(\mathrm{C}_{27}+\mathrm{C}_{29}+\mathrm{C}_{31}\right) /\left(\mathrm{C}_{15}+\mathrm{C}_{17}+\mathrm{C}_{19}\right)
$$

Para evaluar la posible fuente de origen, Fichen et al. ${ }^{14}$ desarrollaron la relación Paq (relación abundancia de cadenas producida por 
plantas de agua dulce flotante sobre cadenas producida por plantas terrestres), expresada como:

$$
\mathrm{Paq}=\left(n-\mathrm{C}_{23}+n-\mathrm{C}_{25}\right) /\left(n-\mathrm{C}_{27}+n-\mathrm{C}_{29}+n-\mathrm{C}_{31}\right)
$$

donde: $\mathrm{Paq}<0,4$ significa entrada de hidrocarburos de origen terrestre. Paq $>0,75$ significa contribución primaria de plantas flotantes. $0,4<$ Paq $<0,75$ significa contribución de origen terrestre y plantas flotantes.

Para determinar el origen de los HAPs uno de los criterios desarrollados corresponde a la razón Fluoranteno/Pireno (Flu/Py), ${ }^{15}$ como una forma de discriminar entre la procedencia petrogénica o pirolítica. La predominancia de aromáticos de alto peso molecular indica que los HAPs son de origen pirogénicos. ${ }^{16}$

El objetivo de este estudio fue evaluar el contenido y distribución de hidrocarburos alifáticos y aromáticos en sedimentos superficiales y de fondo de tres lagunas urbanas para constituir una línea base de los hidrocarburos identificados.

\section{PARTE EXPERIMENTAL}

\section{Área de estudio}

El área de estudio corresponde a tres sistemas lacustre ubicados en el radio urbano de la ciudad de Concepción - Chile; la laguna Lo Galindo (LLG) ubicada a una Latitud $36^{\circ} 48^{\prime} 01^{\prime \prime} \mathrm{S}$ y a una Longitud 7302’31” O, la laguna Lo Méndez (LLM) geográficamente ubicada a una Latitud $36^{\circ} 48^{\prime} 03^{\prime}$ 'S y a una Longitud $73^{\circ} 03^{\prime} 03^{\prime}$ ' O, y la laguna Tres Pascualas (LTP) y una ubicación geográfica de Latitud $36^{\circ} 48^{\prime} 50^{\prime \prime}$ S y Longitud $73^{\circ} 02^{\prime} 30^{\prime \prime} \mathrm{O}$.

El muestreo de las tres lagunas se realizó en Octubre de 2008. Se colectaron muestras de sedimento superficial y de fondo. En cada laguna se establecieron 4 estaciones de muestreo (E1, E2, E3, E4) distribuidas longitudinalmente en las lagunas. Las muestras de sedimento superficial y de núcleos se recolectaron con una draga Eckman de $0,1 \mathrm{~m}^{2}$. Las muestras de sedimento superficial fueron guardadas en bolsas de polietileno y congeladas a $-4{ }^{\circ} \mathrm{C}$ para su posterior análisis. Para la colección de muestra de núcleos, el sedimento se submuestreó con tres testigos de polivinil cloruro (PVC) de $6 \mathrm{~cm}$ de diámetro y 30 $\mathrm{cm}$ de longitud y se almacenaron herméticamente a $-4{ }^{\circ} \mathrm{C}$.

\section{Reactivos}

Se usó agua desionizada calidad ultra pura de resistividad 18,2 $\mathrm{M} \Omega \mathrm{cm}$ (sistema Milli Q, Millipore, Bedford MA). Los reactivos utilizados fueron todos de calidad suprapur Merck (Darmstadt, Alemania).

\section{Análisis de muestra}

Para el procedimiento analítico a las muestras de sedimento descongeladas y pulverizadas se les realizó extracción con diclorometano/hexano (1:1) por ultrasonicación (Branson B3510 Ultrasonic Cleaner) durante un periodo de tiempo de $15 \mathrm{~min}$. El extracto filtrado fue concentrado bajo flujo de nitrógeno ultra puro (grado electrónico 5.0) hasta el volumen requerido para el análisis. El análisis se hizo en un cromatógrafo de gases acoplado a un espectrómetro de masas GCMS QP-5050A, Shimadzu. Las condiciones fueron: temperatura de inyector (modo split razón $1: 10$ ) $325^{\circ} \mathrm{C}$, temperatura de interface $325^{\circ} \mathrm{C}$ y flujo de $\mathrm{He} 1,0 \mathrm{~mL} \mathrm{~min}{ }^{-1}$. Los compuestos fueron separados por medio de una columna cromatográfica DB-5ms (30 m x 0,25 $\mathrm{mm} \times 0,25 \mu \mathrm{m}$ ) de Agilent, y una rampa de temperatura de 40 a 340 ${ }^{\circ} \mathrm{C}$ gradiente de $10{ }^{\circ} \mathrm{C} \mathrm{min}^{-1}$. Las fracciones de hidrocarburos fueron analizadas por el método selección de iones monitoreados (SIM). La determinación y cuantificación de los hidrocarburos se realizó por medio de curvas de calibrado utilizando como estándar una mezcla de hidrocarburos alifáticos de $n-\mathrm{C}_{10}$ a $n-\mathrm{C}_{40},(\mathrm{~S}-4110-100-\mathrm{Y}$ even and uneven + Pristane and Phytane, Chiron AS) y para la cuantificación de los HAPs se usó el estándar MIX 10 PAHs (20 a $\left.1000 \mu \mathrm{g} \mathrm{mL}^{-1}\right)$ de Sigma, Aldrich.

\section{Materia orgánica total, pH y \% de humedad en el sedimento}

La determinación del contenido total de carbón orgánico se realizó a través de la oxidación húmeda de Walkley and Black ${ }^{17}$ y modificado por Jackson. ${ }^{18} \mathrm{El} \mathrm{pH}$ en el sedimento se midió mediante un $\mathrm{pH}$-metro marca Gondo modelo PP-206 WTech. La humedad de sedimento se determinó gravimétricamente y se expresó como \% de humedad.

\section{Análisis estadístico de datos}

Para verificar la existencia de diferencias estadísticamente significativas de los valores medios de factores (CPI, MOT y concentración de HAPs e hidrocarburos alifáticos), se realizó un Análisis de Varianza (ANOVA) de comparación de medias para cada factor en sedimentos de fondo y superficiales para las tres lagunas, con una confianza de un 95\%. Para cada ANOVA se verificaron los tres supuestos requeridos: que los residuos de los factores posean distribución normal con media cero, el supuesto de homocedasticidad (residuos de los factores deben tener la misma varianza entre las lagunas) y que los residuos de los factores sean independientes entre sí. Todos los residuos de los factores cumplieron con el supuesto de independencia, las variables que no cumplieron con los supuestos de normalidad fueron sometidas a una transformación lineal, la cual cumpliese con ese supuesto. Por último, los que no cumplieron el supuesto de homocedasticidad, fueron analizados con la "Prueba Robusta" de comparación de medias de Welch con un 95\% de confianza. Los factores que presentaron diferencias estadísticamente significativas mediante la ANOVA, fueron analizados con el método LSD de Fisher (con un 95\% de confianza), el cual indica qué diferencias entre las medias de las lagunas son estadísticamente significativas. Con este análisis es posible inferir o tener alguna idea de cuales lagunas difieren de las demás. Para los casos en que se cumplió el supuesto de homocedasticidad, se utilizó el programa STATGRAPHICS Plus 5.1 y para los que no se cumplió supuesto, se recurrió al programa estadístico IBM SPSS Statistics 19.

\section{RESULTADOS Y DISCUSIÓN}

Las LLG, LLM y LTP presentaron un promedio de temperatura del agua de 19,$4 ; 19,5$ y $17,1^{\circ} \mathrm{C}$ respectivamente, similar a la temperatura ambiente debido a lo somero de los sistemas. La profundidad, en promedio, fue de 2,8 $\mathrm{m}$ para LLG; 4,6 $\mathrm{m}$ para LLM; y 5,3 m para LTP. El promedio de carbón orgánico más alto lo presenta la LTP con un valor promedio de 4,95\%, (Tabla 1), valor comparable al obtenido en estudio de sedimentos limosos-arcillosos de la laguna Sontecomapan (México). ${ }^{19}$

En todas las muestras se obtuvo la misma distribución de $n$ -alcanos resueltos de $n$-parafinas que van del $n-\mathrm{C}_{14}$ al $n-\mathrm{C}_{31}$ y todas ellas con una predominancia impar, con alta abundancia tanto de cadena corta de $n$-alcanos como de cadena larga. En ninguna muestra se obtuvo aporte de mezcla compleja no resuelta (MCNR).

Los promedios de las concentraciones de $n$-alcanos obtenidos en sedimentos de fondo y superficiales de las lagunas estudiadas se muestran en la Figura 1. El ranking de concentración de $n$-alcanos obtenido fue LLG > LLM > LTP para sedimentos de fondo y LTP > LLG > LLM superficiales. Además se muestra que la concentración 
Tabla 1. Porcentaje de carbono orgánico, materia orgánica total (MOT), Índice de Preferencia de Carbón (CPI) y Predominancia Impar-Par (OEP) en sedimentos superficiales y de fondo de las LLG, LLM y LTP

\begin{tabular}{|c|c|c|c|c|c|}
\hline \multicolumn{6}{|c|}{ Laguna Lo Galindo } \\
\hline Estaciones & Estrato & CPI & OEP & $\begin{array}{c}\text { Carbono orgánico } \\
(\%)\end{array}$ & $\begin{array}{l}\text { MOT }^{1} \\
(\%)\end{array}$ \\
\hline \multirow[t]{2}{*}{$\overline{\mathrm{E} 1}$} & $0-5 \mathrm{~cm}$ & 2,33 & 3,98 & 3,20 & 5,52 \\
\hline & $10-15 \mathrm{~cm}$ & 1,19 & 2,06 & 4,83 & 8,33 \\
\hline \multirow[t]{2}{*}{ E2 } & $0-5 \mathrm{~cm}$ & 2,45 & 2,82 & 3,70 & 6,38 \\
\hline & $10-15 \mathrm{~cm}$ & 2,07 & 1,34 & 3,95 & 6,81 \\
\hline \multirow[t]{2}{*}{ E3 } & $0-5 \mathrm{~cm}$ & 2,34 & 3,72 & 4,85 & 8,36 \\
\hline & $10-15 \mathrm{~cm}$ & 1,50 & 1,97 & 4,78 & 8,24 \\
\hline \multirow[t]{2}{*}{ E4 } & $0-5 \mathrm{~cm}$ & 2,19 & 3,46 & 5,45 & 9,39 \\
\hline & $10-15 \mathrm{~cm}$ & 2,01 & 3,37 & 3,51 & 6,05 \\
\hline \multicolumn{6}{|c|}{ Laguna Lo Méndez } \\
\hline Estaciones & Estrato & CPI & OEP & $\begin{array}{c}\text { Carbono orgánico } \\
(\%)\end{array}$ & $\operatorname{MOT}^{1}(\%)$ \\
\hline \multirow[t]{2}{*}{ E1 } & $0-5 \mathrm{~cm}$ & 3,16 & 4,34 & 3,60 & 6,21 \\
\hline & $10-15 \mathrm{~cm}$ & 2,16 & 3,26 & 5,50 & 9,49 \\
\hline \multirow[t]{2}{*}{ E2 } & $0-5 \mathrm{~cm}$ & 2,14 & 2,22 & 2,75 & 4,74 \\
\hline & $10-15 \mathrm{~cm}$ & 1,89 & 3,26 & 1,88 & 3,24 \\
\hline \multirow[t]{2}{*}{ E3 } & $0-5 \mathrm{~cm}$ & 1,81 & 2,82 & 2,40 & 4,14 \\
\hline & $10-15 \mathrm{~cm}$ & 1,66 & 1,66 & 2,31 & 3,98 \\
\hline \multirow[t]{2}{*}{ E4 } & $0-5 \mathrm{~cm}$ & 1,86 & 3,68 & 2,30 & 3,96 \\
\hline & $10-15 \mathrm{~cm}$ & 1,75 & 2,77 & 1,97 & 3,38 \\
\hline \multicolumn{6}{|c|}{ Laguna Tres Pascualas } \\
\hline Estaciones & Estrato & CPI & OEP & $\begin{array}{c}\text { Carbono orgánico } \\
(\%)\end{array}$ & $\begin{array}{c}\text { MOT }^{1} \\
(\%)\end{array}$ \\
\hline \multirow[t]{2}{*}{ E1 } & $0-5 \mathrm{~cm}$ & 1,67 & 2,55 & 5,20 & 8,96 \\
\hline & $10-15 \mathrm{~cm}$ & 1,98 & 0,99 & 5,94 & 10,25 \\
\hline \multirow[t]{2}{*}{ E2 } & $0-5 \mathrm{~cm}$ & 1,75 & 3,33 & 5,30 & 9,14 \\
\hline & $10-15 \mathrm{~cm}$ & 2,01 & 0,76 & 2,89 & 4,99 \\
\hline \multirow[t]{2}{*}{ E3 } & $0-5 \mathrm{~cm}$ & 1,40 & 3,15 & 4,70 & 8,10 \\
\hline & $10-15 \mathrm{~cm}$ & 1,46 & 1,02 & 4,57 & 7,89 \\
\hline \multirow[t]{2}{*}{ E4 } & $0-5 \mathrm{~cm}$ & 1,62 & 2,74 & 4,60 & 7,93 \\
\hline & $10-15 \mathrm{~cm}$ & 1,03 & 0,77 & 2,82 & 4,86 \\
\hline
\end{tabular}

${ }^{1}$ Materia orgánica total.

de $n$-alcanos de sedimentos de fondo es mayor que en los sedimentos superficiales (la LTP es la que difiere en menor medida) y en las tres lagunas los $n$-alcanos más abundantes son los isoprenoides Pristano (Pris) y Fitano (Fit) en todas las muestras estudiadas, siendo la LTP la laguna con la concentración más alta de estos $n$-alcanos.

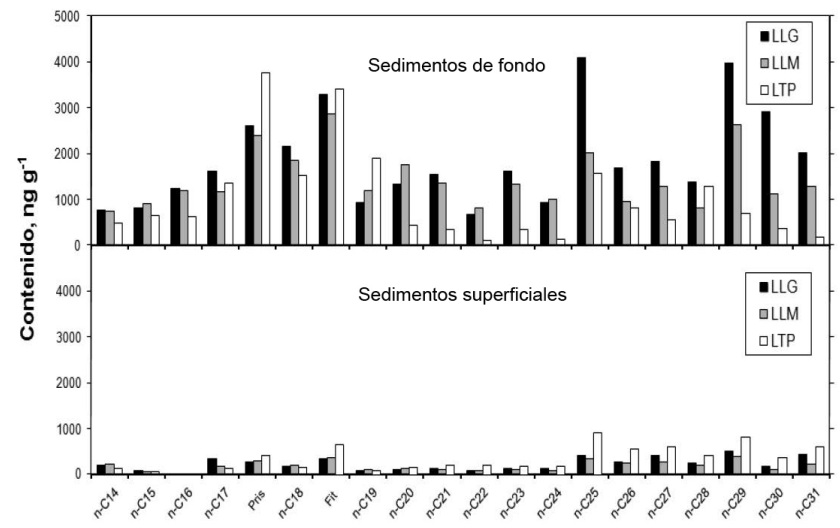

Figura 1. Concentraciones de n-alcanos para sedimentos de fondo y sedimentos superficiales de LLG, LLM y LTP (Fit: Fitano y Pris: Pristano)
La Tabla 2 presenta los factores con diferencia estadísticamente significativa entre sus medias con respecto a las lagunas. Cabe mencionar que todos los HAPs, además del CPI y la MOT, tanto para sedimentos de fondo como superficiales, presentan alguna diferencia significativa entre las lagunas. Por otro lado, todos los hidrocarburos alifáticos, a excepción del $n-\mathrm{C}_{30}$, no presentan ninguna diferencia significativa entre las lagunas para los sedimentos superficiales, por consiguiente, ninguna laguna difiere en cuanto a la concentración de estos hidrocarburos. Mediante el método LSD de Fisher se pudieron analizar todos los factores que presentaron por lo menos una diferencia significativa entre las lagunas, tanto en los sedimentos de fondo como en los superficiales. Con este análisis es posible inferir o tener alguna idea de cuáles son las lagunas que difieren del resto. Se obtuvo que LLM y LTP difieren de forma inferior a las otras lagunas en el porcentaje de MOT y CPI respectivamente, tanto para los sedimentos de fondo como para los superficiales. Por otro lado, las concentraciones de los hidrocarburos de cadenas largas de alcanos $\left(n-\mathrm{C}_{27}\right.$ al $\left.n-\mathrm{C}_{31}\right)$ en los sedimentos de fondo tienden a ser mayor en la LLM. Finalmente, las concentraciones de Flu y Py difieren de forma mayor en LLM en los sedimentos de fondo, mientras que para las concentraciones de éstos en los sedimentos superficiales, la LTP es la que difiere de forma mayor.

Los gráficos de "Caja y Bigotes" de concentraciones de hidrocarburos alifáticos (Figura 1S, material suplementario) muestran que los datos tienden a concentrarse hacia la parte inferior de la distribución, por lo tanto se podría inferir que los datos poseen una distribución asimétrica positiva. Además en ambas figuras se observa que la LLM es la que tiene menor dispersión de los datos con respecto a las otras dos lagunas. En la Figura 2S, material suplementario, se muestran los gráficos de "Caja y Bigotes" de las concentraciones de los HAPs para cada estación de los sedimentos de fondo. Se observa que para las LLG y LLM, los datos tienden a concentrarse hacia la parte superior de la distribución (excepto la E2 de la LLG), por lo que podría inferirse que para estas dos lagunas los datos poseen una distribución asimétrica negativa. Además, a diferencia de los datos de hidrocarburos alifáticos, la LLM es la que posee mayor dispersión de los valores.

Para las tres lagunas estudiadas, se obtuvo valores de LMW/ HMW $<1$ para sedimentos superficiales y de fondo; los valores de $\Sigma$ $n$-alcanos $/ n-\mathrm{C}_{16}$ fueron $>50$ en sedimentos superficiales, y entre $15 \mathrm{y}$ 50 para los de fondo, lo que indica un aporte biogénico proveniente de plantas. Para evaluar la potencial contribución de las plantas terrestres a la materia orgánica Peters et al. ${ }^{20}$ sugieren el uso del índice CPI. Basado en el cálculo de este índice, en los tres sistemas estudiados se obtuvieron valores que variaron en un rango muy estrecho y una disminución desde la superficie hacia la profundidad.

En literatura ${ }^{21}$ se propone el uso de la relación Pris/Fit como proxy para el nivel de oxidación en los sedimentos, valores bajos indican deposición bajo procesos aeróbicos. Para los sedimentos estudiados de las tres lagunas, los valores de la relación Pris/Fit obtenidos son similares entre sí (Figura 3S, material suplementario). Se obtuvo para la LTP una relación Pris/Fit de tendencia creciente con la profundidad, lo que indica que en este sistema hay un cambio en el nivel de oxígeno. Además ésta presenta mayores valores de las relaciones Pris $/ n-\mathrm{C}_{17} \mathrm{y}$ $\mathrm{Fit} / n-\mathrm{C}_{18}$ con respecto a las otras dos lagunas estudiadas. Los valores de la relación Pris/Fit obtenidos son similares a los encontrados por otros investigadores en estudios de lagos y ríos de Polonia, ${ }^{22}$ Japón ${ }^{23}$ y España. ${ }^{24}$ La predominancia de estos isoprenos indica una fuente biogénica, ${ }^{25}$ lo que se confirma con los valores obtenidos en Tabla 1, de los índices CPI y OEP, ya que las cadenas impar tienden a prevalecer en los materiales biológicos. ${ }^{26}$

En Tabla 3 se muestran los indicadores de origen de hidrocarburos en sedimentos de fondo y superficiales. Los valores del índice TAR indican que el origen de hidrocarburos para las lagunas LLG y LLM 
Tabla 2. Resumen del Análisis de Varianza (ANOVA) para sedimentos de fondo y superficiales de LLG, LLM y LTP, con un nivel de 95\% de confianza

\begin{tabular}{|c|c|c|c|c|c|}
\hline & Factores & $\mathrm{F}$ & $\mathrm{F}_{\text {Critico }}$ & Valor-p & $\begin{array}{c}\text { Nivel de Signifi- } \\
\text { cancia }\end{array}$ \\
\hline \multirow{27}{*}{$\begin{array}{l}0 \\
0 \\
0 \\
0 \\
0 \\
0 \\
0 \\
0 \\
0 \\
0 \\
0 \\
0\end{array}$} & Phe & 8,096 & 4,26 & 0,009736 & Significativo \\
\hline & Flu & 23,216 & 4,26 & 0,00028 & Significativo \\
\hline & Py & 19,445 & 4,26 & 0,000541 & Significativo \\
\hline & $\mathrm{BbF}$ & 6,152 & 4,26 & 0,0207 & Significativo \\
\hline & $\mathrm{BkF}$ & 4,933 & 4,26 & 0,035768 & Significativo \\
\hline & $n-\mathrm{C}_{14}$ & 1,917 & 4,26 & 0,202504 & No significativo \\
\hline & $n-\mathrm{C}_{15}$ & 0,777 & 4,26 & 0,488458 & No significativo \\
\hline & $n-\mathrm{C}_{16}$ & 3,677 & 4,26 & 0,068038 & No significativo \\
\hline & $n-\mathrm{C}_{17}$ & 0,29 & 4,26 & 0,754688 & No significativo \\
\hline & Pris & 0,571 & 4,26 & 0,584112 & No significativo \\
\hline & $n-\mathrm{C}_{18}$ & 0,094 & 4,26 & 0,910761 & No significativo \\
\hline & Fit & 0,951 & 4,26 & 0,421895 & No significativo \\
\hline & $n-\mathrm{C}_{19} *$ & - & - & 0,276488 & No significativo \\
\hline & $n-\mathrm{C}_{20}$ & 3,297 & 4,26 & 0,084314 & No significativo \\
\hline & $n-\mathrm{C}_{21}$ & 11,664 & 4,26 & 0,00317 & Significativo \\
\hline & $n-\mathrm{C}_{22}$ & 2,75 & 4,26 & 0,116935 & No significativo \\
\hline & $n-\mathrm{C}_{23}$ & 13,687 & 4,26 & 0,001864 & Significativo \\
\hline & $n-\mathrm{C}_{24}$ & 2,816 & 4,26 & 0,112287 & No significativo \\
\hline & $n-\mathrm{C}_{25}$ & 4,314 & 4,26 & 0,048558 & Significativo \\
\hline & $n-\mathrm{C}_{26}$ & 7,643 & 4,26 & 0,011481 & Significativo \\
\hline & $n-\mathrm{C}_{27}$ & 10,701 & 4,26 & 0,004179 & Significativo \\
\hline & $n-\mathrm{C}_{28}$ & 64,149 & 4,26 & 0,000005 & Significativo \\
\hline & $n-\mathrm{C}_{29}$ & 7,092 & 4,26 & 0,014148 & Significativo \\
\hline & $n-\mathrm{C}_{30}$ & 6,131 & 4,26 & 0,020887 & Significativo \\
\hline & $n-\mathrm{C}_{31}$ & 6,465 & 4,26 & 0,01817 & Significativo \\
\hline & CPI & 4,298 & 4,26 & 0,04894 & Significativo \\
\hline & MOT & 9,86 & 4,26 & 0,005398 & Significativo \\
\hline \multirow{23}{*}{ 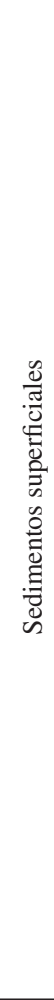 } & Flu & 62,591 & 4,46 & 0,000013 & Significativo \\
\hline & Py & 63,435 & 4,46 & 0,000012 & Significativo \\
\hline & $n-\mathrm{C}_{14}$ & 0,951 & 4,46 & 0,426067 & No Significativo \\
\hline & $n-\mathrm{C}_{15}$ & 3,506 & 4,26 & 0,074847 & No Significativo \\
\hline & $n-\mathrm{C}_{17}$ & 1,111 & 4,26 & 0,370583 & No Significativo \\
\hline & Pris & 1,181 & 4,26 & 0,350249 & No Significativo \\
\hline & $n-\mathrm{C}_{18}$ & 0,058 & 4,26 & 0,944216 & No Significativo \\
\hline & Fit & 2,237 & 4,26 & 0,162642 & No Significativo \\
\hline & $n-\mathrm{C}_{19}$ & 0,177 & 4,26 & 0,840996 & No Significativo \\
\hline & $n-\mathrm{C}_{20}$ & 0,37 & 4,26 & 0,700503 & No Significativo \\
\hline & $n-\mathrm{C}_{21}$ & 1,287 & 4,26 & 0,322318 & No Significativo \\
\hline & $n-\mathrm{C}_{22}$ & 1,003 & 4,46 & 0,408725 & No Significativo \\
\hline & $n-\mathrm{C}_{23}$ & 0,952 & 4,26 & 0,421653 & No Significativo \\
\hline & $n-\mathrm{C}_{24}$ & 0,539 & 4,74 & 0,605606 & No Significativo \\
\hline & $n-\mathrm{C}_{25} *$ & - & - & 0,337574 & No Significativo \\
\hline & $n-\mathrm{C}_{26}$ & 2,317 & 4,26 & 0,154258 & No Significativo \\
\hline & $n-\mathrm{C}_{27}$ & 2,082 & 4,26 & 0,18066 & No Significativo \\
\hline & $n-\mathrm{C}_{28}{ }^{*}$ & - & - & 0,227039 & No Significativo \\
\hline & $n-\mathrm{C}_{29} *$ & - & - & 0,257451 & No Significativo \\
\hline & $n-\mathrm{C}_{30}$ & 5,194 & 4,26 & 0,031633 & Significativo \\
\hline & $n-\mathrm{C}_{31}$ & 2,8 & 4,26 & 0,113396 & No Significativo \\
\hline & CPI & 4,298 & 4,26 & 0,04894 & Significativo \\
\hline & MOT & 9,86 & 4,26 & 0,005398 & Significativo \\
\hline
\end{tabular}

*Factores analizados por la "Prueba Robusta" de Welch. es acuático tanto para los sedimentos superficiales como los de fondo. En cambio para LTP la fuente de origen es una mezcla entre terrestre y acuático. Aunque la distribución de $n$-alcanos obtenida con valores de CPI, OEP y relación Pris/Fit (Tabla 1), indican predominio de hidrocarburos de origen biogénico, la presencia de cadenas cortas sugiere una contribución distinta a la de las plantas terrestres. Estas cadenas se asocian al aporte de algas y/o macrófitas. ${ }^{27}$ Por otro lado, los valores del índice Paq indican que para la LLG y LLM se obtienen valores similares con una contribución mezcla de origen terrestre y acuático. En cambio, para la LTP se observa mayor contribución de plantas flotantes; lo que concuerda con el grave problema de eutrificación que presenta este sistema acuícola urbano.

Tabla 3. Indicadores de orígenes de hidrocarburos para LLG, LLM y LTP

\begin{tabular}{|c|c|c|c|}
\hline \multicolumn{4}{|c|}{ Sedimentos de fondo } \\
\hline & LLG & LLM & LTP \\
\hline Plancton $\left(\right.$ ng g $\left.^{-1}\right)$ & 3,34 & 3,27 & 3,9 \\
\hline \multicolumn{4}{|l|}{$\left(\mathrm{C}_{15}+\mathrm{C}_{17}+\mathrm{C}_{19}\right)$} \\
\hline Plantas terrestres $\left(\mathrm{ng} \mathrm{g}^{-1}\right)$ & 9,89 & 5,94 & 2,79 \\
\hline \multicolumn{4}{|l|}{$\left(\mathrm{C}_{25}+\mathrm{C}_{22}+\mathrm{C}_{29}\right)$} \\
\hline Indicador de petróleo (1) $\left(\mathrm{ng} \mathrm{g}^{-1}\right)$ & 4,72 & 4,81 & 2,59 \\
\hline \multicolumn{4}{|l|}{$\left(\mathrm{C}_{16}+\mathrm{C}_{18}+\mathrm{C}_{20}\right)$} \\
\hline Indicador de petróleo (2) $\left(\mathrm{ng} \mathrm{g}^{-1}\right)$ & 6,89 & 3,89 & 2,6 \\
\hline \multicolumn{4}{|l|}{$\left(\mathrm{C}_{24}+\mathrm{C}_{26}+\mathrm{C}_{28}+\mathrm{C}_{30}\right)$} \\
\hline TAR $=\left(\mathrm{C}_{27}+\mathrm{C}_{29}+\mathrm{C}_{31}\right) /\left(\mathrm{C}_{15}+\mathrm{C}_{17}+\mathrm{C}_{19}\right)$ & 2,34 & 1,59 & 0,36 \\
\hline Paq $=\left(\mathrm{C}_{23}+\mathrm{C}_{25}\right) /\left(\mathrm{C}_{27}+\mathrm{C}_{29}+\mathrm{C}_{31}\right)$ & 0,73 & 0,64 & 1,35 \\
\hline \multicolumn{4}{|c|}{ Sedimentos superficiales } \\
\hline & LLG & LLM & LTP \\
\hline Plancton (ng g-1) & 0,51 & 0,33 & 0,27 \\
\hline \multicolumn{4}{|l|}{$\left(\mathrm{C}_{15}+\mathrm{C}_{17}+\mathrm{C}_{19}\right)$} \\
\hline Plantas terrestres ( $\mathrm{ng} \mathrm{g}^{-1}$ ) & 1,35 & 1,01 & 2,31 \\
\hline \multicolumn{4}{|l|}{$\left(\mathrm{C}_{25}+\mathrm{C}_{22}+\mathrm{C}_{29}\right)$} \\
\hline Indicador de petróleo (1) $\left(\mathrm{ng} \mathrm{g}^{-1}\right)$ & 0,3 & 0,31 & 0,32 \\
\hline \multicolumn{4}{|l|}{$\left(\mathrm{C}_{16}+\mathrm{C}_{18}+\mathrm{C}_{20}\right)$} \\
\hline Indicador de petróleo (2) $\left(\mathrm{ng} \mathrm{g}^{-1}\right)$ & 0,83 & 0,63 & 1,51 \\
\hline \multicolumn{4}{|l|}{$\left(\mathrm{C}_{24}+\mathrm{C}_{26}+\mathrm{C}_{28}+\mathrm{C}_{30}\right)$} \\
\hline TAR $=\left(\mathrm{C}_{27}+\mathrm{C}_{29}+\mathrm{C}_{31}\right) /\left(\mathrm{C}_{15}+\mathrm{C}_{17}+\mathrm{C}_{19}\right)$ & 2,64 & 2,7 & 7,51 \\
\hline Paq $=\left(\mathrm{C}_{23}+\mathrm{C}_{25}\right) /\left(\mathrm{C}_{27}+\mathrm{C}_{29}+\mathrm{C}_{31}\right)$ & 0,41 & 0,5 & 0,55 \\
\hline
\end{tabular}

TAR: Razón entre material terrígeno a acuático. Paq: Relación abundancia de cadenas producidas por plantas de agua dulce flotantes sobre cadenas producidas por plantas terrestres.

Se compararon los resultados con un trabajo de literatura ${ }^{28}$ de un lugar cercano a las lagunas LLG, LLM y LTP, correspondiente a un estudio paleolimnológico de la laguna Chica de San Pedro (36 50'40" $\left.\mathrm{S} ; 7^{\circ} 05^{\prime} 15^{\prime \prime} \mathrm{O}\right)$. Se informan al igual que en las LLG, LLM y LTP una importante presencia de los $n$-alcanos $n-\mathrm{C}_{27}, n-\mathrm{C}_{29}$ y $n$ - $\mathrm{C}_{31}$. El índice TAR informado por Urrutia et al. ${ }^{28}$ es mayor a 1 y con un valor máximo de 5,4; similar a lo obtenido para sedimentos superficiales de LLG, LLM y LTP. Otro aspecto concordante con los resultados presentados en este trabajo es que las concentraciones de cadena larga son mayores en los sedimentos superficiales que en las capas más profundas y que en el fondo prevalecen las cadenas de $n$-alcanos cortas.

Los HAPs más abundantes fueron los de mayor peso molecular y corresponden a Flu y Py. Su presencia puede relacionarse con las actividades propias del área estudiada y también a procesos de 
combustión. Ruus et al. ${ }^{29}$ en estudios de sedimentos marinos encontraron que, de todos los HAPs, el Py es el que presenta mayor capacidad de bioacumulación. En las tres lagunas se observa presencia de un HAP de tres ciclos que corresponde al Fenantreno (Phe), cuyo origen es petroquímico. ${ }^{30} \mathrm{La}$ presencia de este compuesto probablemente se debe a que durante mucho tiempo estos sistemas lacustres urbanos fueron utilizados como basurales, además de la depositación de partículas secas de la atmósfera por la proximidad de una autopista y de un aeropuerto. En menor concentración se encontraron Benzo (b) Fluoranteno (BbF) y Benzo (k) Fluoranteno (BkF). Estos HAPs pueden ser acumulados en el ambiente. En Figura 2 se comparan las relaciones Flu/Py de las muestras de sedimento superficial con campaña anterior (Enero 2005). Se observa que en todas las lagunas el valor de la relación de estos hidrocarburos aumentó en el segundo muestreo, en particular en la laguna Lo Galindo cuyo valor se duplicó. Esto se puede explicar por el incendio de bosque en el cerro cercano a esta laguna ocurrido en el verano del 2007, ya que una fuente importante de estos HAPs en el ambiente proviene de incendios forestales. ${ }^{31,32}$

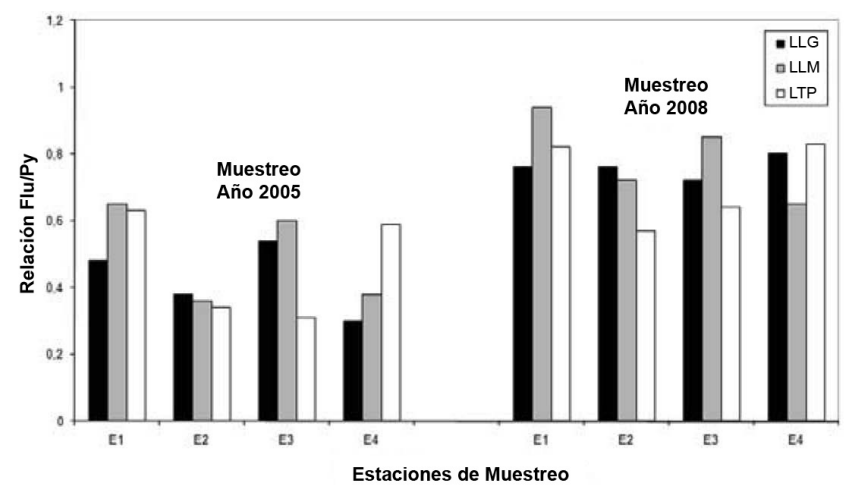

Figura 2. Relación de concentraciones de Fluoranteno/Pireno para los años 2005 y 2008 en sedimentos superficiales de LLG, LLM y LTP

Los HAPs encontrados indican un aporte de compuestos de fuentes pirogénicas, y las diferencias de éstos entre las lagunas sugieren que se debe a emisiones diferentes, ${ }^{6}$ debidas a quema de material vegetal o quema de combustibles fósiles. Para diferenciar entre estas fuentes pirogénicas, se determinó la relación Flu/Py, y la relación $\mathrm{Flu} /(\mathrm{Flu}+\mathrm{Py})$ para diferenciar entre HAPs de origen petroquímico y pirogénico. En Tabla 4 se muestran los valores de las relaciones Flu/ Py con valores similares para las tres lagunas estudiadas y todos $<$ 1 y los valores de las relaciones Flu/(Flu+Py) $<0,5$ que son indicativos de contaminación de origen pirolítico,${ }^{33}$ tanto proveniente de combustibles fósiles usados en transporte automovilístico, como de la quema producida por incendios forestales. Cabe señalar que el contenido de Flu y Py aumenta desde la superficie hacia el fondo. El Flu se encuentra más en el fondo posiblemente debido a la migración de los HAPs más solubles asociados a partículas finas y su posterior acumulación.

\section{CONCLUSIONES}

De los tres sistemas lagunares estudiados la LTP presenta la concentración más alta de $n$-alcanos para sedimentos superficiales, lo que concuerda con el grave problema de eutrofización de este sistema, mientras que la LLG presenta las mayores concentraciones para sedimentos de fondo. Según los valores de los índices aplicados se puede inferir que el origen de los hidrocarburos en las lagunas estudiadas es biogénico.

La concentración más alta de HAPs en sedimentos de fondo
Tabla 4. Concentraciones de HAPs en $\mathrm{ng} \mathrm{g}^{-1}$, suma de HAPs e Indicadores de orígenes de HAPs para LLG, LLM y LTP

\begin{tabular}{|c|c|c|c|}
\hline \multicolumn{4}{|c|}{ Sedimentos de fondo } \\
\hline & LLG & LLM & LTP \\
\hline Phe $\left(\right.$ ng g $\left.^{-1}\right)$ & 106,1 & 171,3 & 121,4 \\
\hline Flu (ng g $\left.{ }^{-1}\right)$ & 103,4 & 194,8 & 153,3 \\
\hline Py $\left(\operatorname{ng~g}^{-1}\right)$ & 154 & 218,0 & 191,3 \\
\hline $\mathrm{BbF}\left(\mathrm{ng} \mathrm{g}^{-1}\right)$ & 36,4 & 39,6 & 46,5 \\
\hline $\mathrm{BkF}\left(\mathrm{ng} \mathrm{g}^{-1}\right)$ & 29,7 & 28,1 & 32,7 \\
\hline$\Sigma$ HAPs $\left(\right.$ ng g $\left.^{-1}\right)$ & 429,6 & 651,8 & 545,2 \\
\hline Flu/Py & 0,66 & 0,68 & 0,66 \\
\hline $\mathrm{Flu} /(\mathrm{Flu}+\mathrm{Py})$ & 0,40 & 0,40 & 0,398 \\
\hline \multicolumn{4}{|c|}{ Sedimentos superficiales } \\
\hline & LLG & LLM & LTP \\
\hline Phe $\left(\right.$ ng g $\left.^{-1}\right)$ & nd & nd & nd \\
\hline Flu $\left(\mathrm{ng} \mathrm{g}^{-1}\right)$ & 22,2 & 19,1 & 57,5 \\
\hline Py $\left(\operatorname{ng~g}^{-1}\right)$ & 33,3 & 28,4 & 86,9 \\
\hline $\mathrm{BbF}\left(\mathrm{ng} \mathrm{g}^{-1}\right)$ & nd & nd & nd \\
\hline $\mathrm{BkF}\left(\mathrm{ng} \mathrm{g}^{-1}\right)$ & nd & nd & nd \\
\hline$\Sigma$ HAPs $\left(\right.$ ng g $\left.^{-1}\right)$ & 55,5 & 47,5 & 144,4 \\
\hline $\mathrm{Flu} / \mathrm{Py}$ & 0,87 & 0,86 & 0,79 \\
\hline Flu/(Flu+Py) & 0,46 & 0,46 & 0,44 \\
\hline
\end{tabular}

corresponde a la LLM y en sedimentos superficiales a la LTP. En las tres lagunas estudiadas los HAPs más abundantes encontrados son Flu y Py, y de acuerdo al valor de las relaciones obtenidas de Flu/Py se concluye que el origen de los HAPs es principalmente pirolítico, tanto proveniente de combustibles fósiles (gasolinas, aceites y lubricantes) usados en transporte automovilístico, como de la quema producida por incendios forestales.

La recopilación y análisis de los datos de los diferentes contenidos de hidrocarburos constituye una importante línea base de registro que puede servir de ayuda para determinar el grado de impacto ecológico como también para acordar acciones futuras de manejo y uso de zonas lagunares urbanas.

\section{MATERIAL SUPLEMENTARIO}

Disponible en http://quimicanova.sbq.org.br, en forma de archivo PDF y con acceso libre. En Figura 1S se presentan los gráficos de "Caja y Bigotes" para las concentraciones (ng g ${ }^{-1}$ ) de los Hidrocarburos Alifáticos en las estaciones LLG, LLM y LTP: a) Sedimentos de fondo y b) Sedimentos superficiales. La Figura $2 \mathrm{~S}$ se presenta el gráfico de "Caja y Bigotes" para las concentraciones (ng g-1) de los Hidrocarburos Aromáticos Policíclicos en los sedimentos de fondo de las estaciones LLG, LLM y LTP. La Figura 3S muestra los parámetros geoquímico orgánicos (promedio) de Hidrocarburos Alifáticos en sedimentos de fondo y superficial de la LLG, LLM y LTP.

\section{AGRADECIMIENTOS}

Los autores agradecen a la Facultad de Ciencias de la Universidad Católica de la Santísima Concepción y especial agradecimiento por el aporte financiero a la Dirección de Investigación de la Universidad Católica de la Santísima Concepción a través del proyecto DIN 0610. Agradecen también el apoyo al proyecto Fondecyt $N^{\circ} 1120805$. 


\section{REFERENCIAS}

1. Serrazanetti, G. P.; Conte, L. S.; Carpené, E.; Bergami, C.; FondaUmani, S.; Chemosphere 1991, 23, 925.

2. Conde, D.; Arocena, R.; Rodríguez-Gallego, L.; AMBIOS 2002, 5, 32.

3. González, E.; Cifuentes, M.; Medina, V.; Ahumada, R.; Neira, J.; Quim. Nova 2009, 32, 902.

4. Díaz, N.; Alfaro, J.; Garza, H.; Ciencia UANL 2001, 4, 420.

5. Lin, O.; Mendelssohn, A.; Carney, K.; Miles, S.; Bryner, N.; Walton, W.; Environ. Sci. Technol. 2005, 39, 1856.

6. Simoneit, B.; Appl. Geochem. 2002, 17, 129.

7. Pérez, O.; Ruiz, F.; Torres, I.; Beltrán, J.; Rev. Invest. Mar. 2008, 29, 179.

8. Gomes, A. O.; Azevedo, D. A.; J. Braz. Chem. Soc. 2003, 14, 358

9. Medeiros, P. M.; Bícego, M. C.; Mar. Pollut. Bull. 2004, 49, 761.

10. Colombo, J. C.; Pelletier, E.; Brochu, C.; Khalil, M.; Environ. Sci. Technol. 1989, 23, 888.

11. McLaughlin, J.; McLaughlin, R.; Kweller, N.; J. Coal Geol. 1987, 7, 21.

12. Scalan, R.; Smith, J.; Geochim. Cosmochim. Acta 1970, 34, 623.

13. Bourbonniere, R. A.; Meyer, P. A.; Limnology and Oceanography 1996, 41,352 .

14. Ficken, K.; Li, B.; Swan, D.; Eglinton, G.; Org. Chem. 2000, 31, 745.

15. Porte, C.; Barceló, D.; Albaigés, J.; J. Chromatogr. 1998, 442, 386.

16. Kalf, D.; Crommentujin, T.; van Plassche, E.; Ecotoxicol. Environ. Safety 1997, 36, 89.

17. Walkley, A.; Black, I.; Soil Sci. 1946, 63, 251.

18. Jackson, M.; Soil Chemical Analysis, $1^{\text {st }}$ ed., Prentice Hall Pub. Co.: New York, 1958.
19. Calva, L.; Botello, A.; Ponce, G.; Hidrobiología 2005, 15, 97.

20. Peters, K.; Walters, C.; Moldowan, M.; The Biomarker Guide, Cambridge University Press: New York, 2005.

21. Riccardi, C.; Di Filippo, P.; Pomata, D.; Incoronato, F.; Di Basilio, M.; Petrangeli, M.; Spicaglia, S.; Sci. Total Environ. 2008, 393, 50.

22. Bechtel, A.; Woszczyk, M.; Reischenbacher, D.; Sachsenhofer, R. F.; Gratzer, R.; Püttmann, W.; Spychalski, W.; Org. Chem. 2007, 38, 1112.

23. Jeng, W-L.; Huh, C-A.; Estuarine Coastal and Shelf Science 2006, 66, 217.

24. Blanco, C.; Prego, R.; Azpíroz, R.; Fernandez, M.; Ciencias Marinas 2006, 32, 429.

25. Evangelista, A.; Nascimento, I.; Pereira, S.; López, M.; Martins, L.; Fillmann, G.; Ciencias Marinas 2005, 31, 43.

26. Bouloubassi, I.; Fillaux, J.; Saliot, A.; Mar. Pollut. Bull. 2001, 42, 1335.

27. Bechtel, A.; Sachsenhofer, R.; Markic, M.; Gratzer, R.; Lucke, A.; Puttmann, W.; Org. Geochem. 2008, 34, 1277.

28. Urrutia, R.; Sabbe, K.; Cruces, F.; Pozo, K.; Becerra, J.; Rev. Chil. Hist. Nat. 2000, 73, 717.

29. Ruus, A.; Schaanning, M.; Oxnevad, S.; Hylland, K.; Aquat. Toxicol. 2005, 72, 273.

30. Douglas, G.; Bence, A.; Prince, R.; McMillen, S.; Butler, E.; Environ. Sci. Technol. 1996, 30, 2332.

31. Krauss, M.; Wilcke, W.; Zech, W.; Environ. Pollut. 2000, 110, 79.

32. Bucheli, T. D.; Blum, F.; Desaules, A.; Gustafsson, Ö.; Chemosphere 2004, 56, 1061

33. Fernandez, P.; Vilanova, R. M.; Grimalt, J. O.; Environ. Sci. Technol. 1999, 33, 3716 


\section{CONTENIDO, DISTRIBUCIÓN Y ORIGEN DE HIDROCARBUROS EN SEDIMENTOS DE TRES LAGUNAS URBANAS DE CONCEPCIÓN - CHILE}

\section{Elizabeth González Sepúlveda*}

Departamento de Química Ambiental, Facultad de Ciencias, Universidad Católica de la Santísima Concepción, Casilla 297, Concepción, Chile

\section{Rodrigo Loyola Sepúlveda}

Departamento de Oceanografía Química, Universidad de Concepción, Concepción, Chile

José Neira Hinojosa

Departamento de Análisis Instrumental, Facultad de Farmacia, Universidad de Concepción, Concepción, Chile

\section{Felipe Neira González}

Departamento de Ingeniería Industrial, Facultad de Ingeniería, Universidad de Concepción, Concepción, Chile
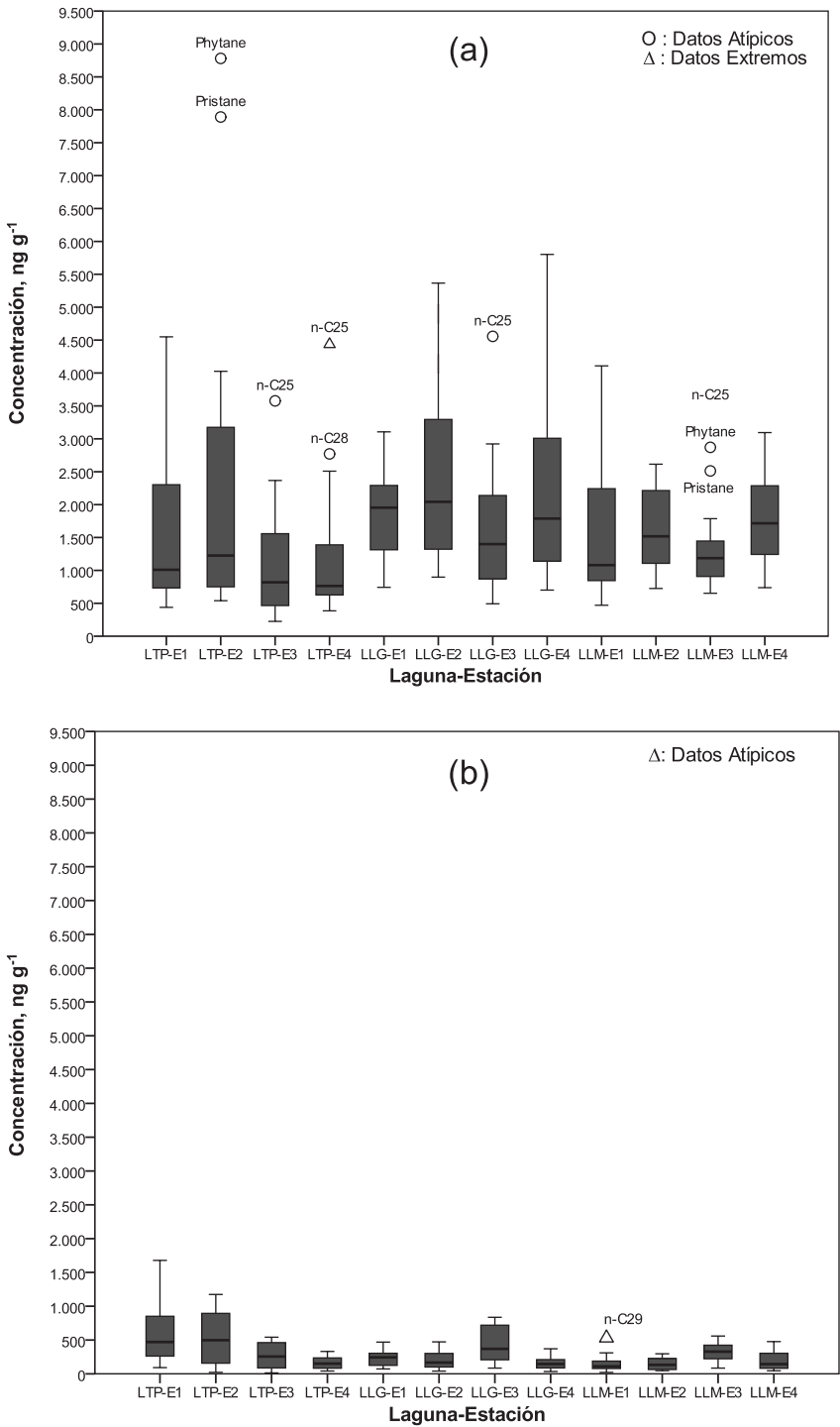

Figura 1S. Gráficos de "Caja y Bigotes" para las concentraciones $\left(n g g^{-1}\right)$ de los Hidrocarburos Alifáticos en las estaciones LLG, LLM y LTP: a) sedimentos de fondo y $b$ ) sedimentos superficiales

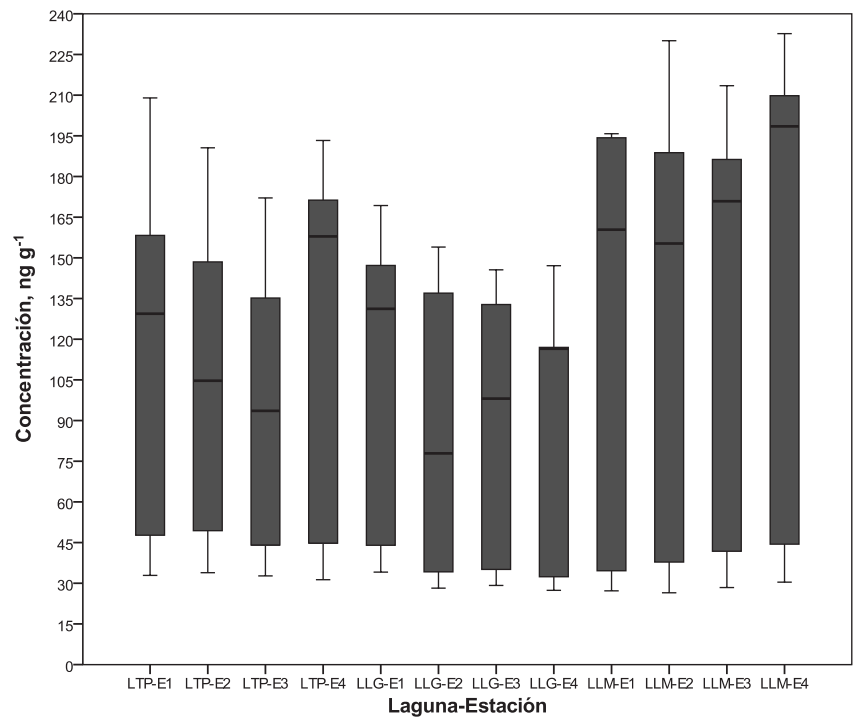

Figura 2S. Gráfico de "Caja y Bigotes" para las concentraciones $\left(n g \mathrm{~g}^{-1}\right)$ de los Hidrocarburos Aromáticos Policíclicos en los sedimentos de fondo de las estaciones $L L G$, LLM y LTP

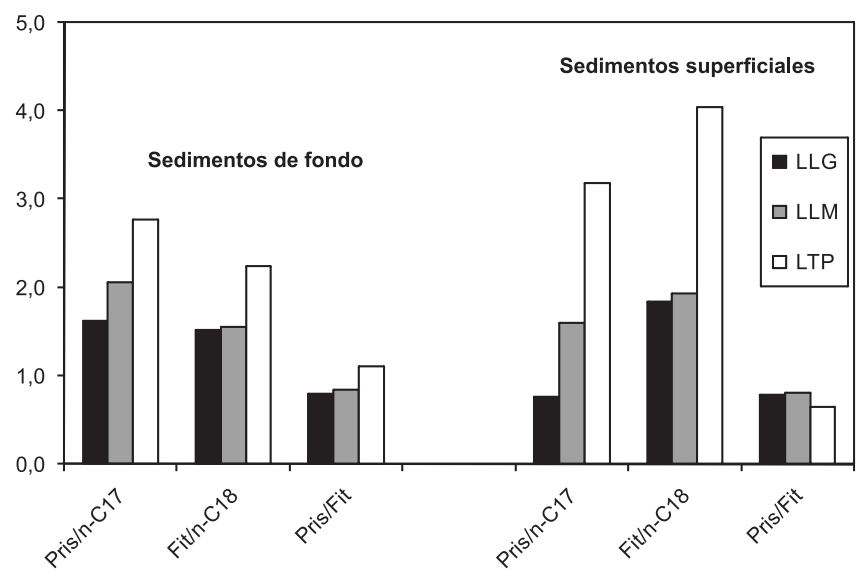

Figura 3S. Parámetros geoquímico orgánicos (promedio) de Hidrocarburos Alifáticos en sedimentos de fondo y superficial de LLG, LLM y LTP 